\title{
Analysis of the Distribution of Exchange-Rates near the 2008 Global Financial Crisis
}

\author{
Doobae Jun ${ }^{1,2}$ \\ Jinsu Kim ${ }^{1}$ \\ Gwangil Kim ${ }^{1,2, ~ * ~}$ \\ ${ }^{1}$ Department of Mathematics, \\ Gyeongsang National University, \\ Jinju, Republic of Korea \\ ${ }^{2}$ Research Institute of Natural Science, \\ Gyeongsang National University, \\ Jinju, Republic of Korea \\ ${ }^{*}$ Corresponding Author
}

DOI: https://doi.org/10.36941/ajis-2020-0073

\begin{abstract}
We search for indicators that might have predicted the 2008 financial crisis, by analyzing the standardized normalized distribution of exchange-rates. We find that this distribution was close to normal during the crisis, but had an exceptionally high kurtosis in the second quarter of 2006, indicating the beginning of longterm USD weakness. Somewhat nearer to the crisis, we can also see suggestive fluctuations in some exchange-rates. Further, we analyze stock-market indices across the crisis, and show that they responded more sensitively than exchange-rates, and that the distribution of stock-market indices also has an exceptional value of kurtosis at Q2 2006, suggesting that the kurtosis of the distribution of exchange-rates might have provided as an early indicator of the crisis.
\end{abstract}

Keywords: 2008 financial crisis, Exchange-rates, Standardization, Standardized normalization, Statistical moments

\section{Introduction}

Many economists (2009) consider the 2008 global financial crisis to have been the worst since the 1930s. Many researchers who take this view have tried to explain the crisis in terms of several economic variables: Blanchard and Faruqee (2010) suggest that levels of trade and financial exposure caused the crisis; Lane and Milesi-Ferretti (2011) suggest that the ratio of private credit to GDP, current account deficits and openness to trade are also key variables. However, there seems to be no robust consensus on the determinants of the crisis or the key indicators of its progress, as Rose and Spiegel (2010, 2012) observe.

In this paper, we use the standardization and normalization methods (2017) to investigate whether currency-side factors responded to the 2008 financial crisis in a way that could have provided indicators that might have been useful in predicting its onset. We consider the world foreign exchange markets as a distribution of standardized and normalized exchange-rates. In 2012, 
Frankel and Saravelos (2012) suggested that the overvaluation of international reserves and real exchange-rates might have been better than other candidate indicators in predicting the 2008 financial crisis. By observing the behavior of the statistical moments of the distribution of standardized exchange-rates obtained from the IMF quarterly figures (2015) for 73 countries (see Figure 1), we will analyze the sensitivity of the currency-side factors during the 2008 financial crisis.

We show that, while these factors had a near-normal distribution during the crisis, a year or so earlier it was abnormal with large values of kurtosis. We additionally observe interesting fluctuations in the behavior of the exchange rates of some currencies closer to the crisis: the USD, HKD and CNY tracked closely each other over the entire period that we observe. In particular, the US dollar was weak for a long time prior to the crisis. By analyzing the distribution of exchange-rates and stockmarket indices, we have found that stock-markets responded more sensitively to the crisis than foreign exchange markets. This conflicts with the view of the suggestion of Frankel and Saravelos. By observing the distribution of values of kurtosis of these two distributions, we can also show that both had unusual values of kurtosis at the beginning of the long period of USD weakness, which might have signaled the genesis of the crisis.

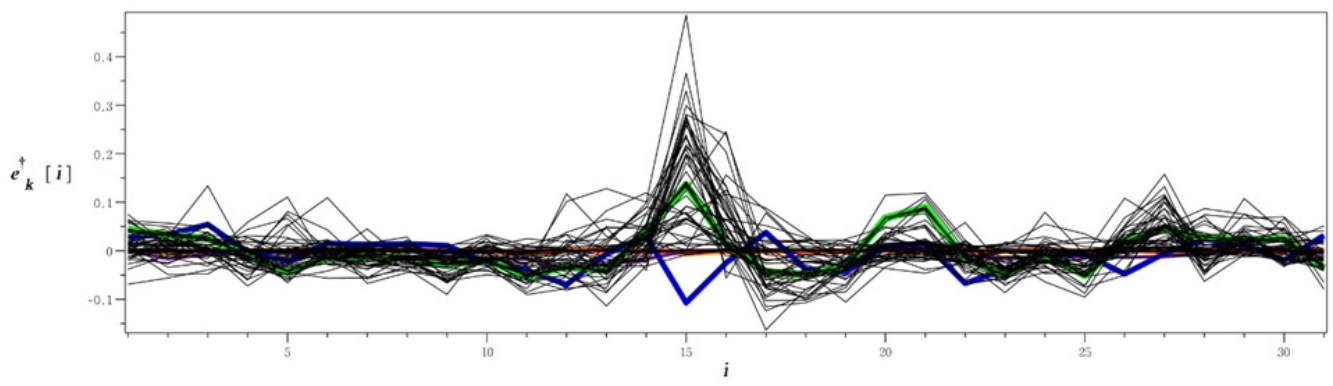

Fig. 1. Standardized exchange-rates over the 31 quarters from $\mathrm{Q}_{1} 2005$ to $\mathrm{Q}_{4}$ 2012: The red, green and blue curves respectively correspond to the USD, EUR and JPY.

\section{Method and Analysis}

\subsection{Standardized exchange-rates and their behavior}

We standardize the IMF exchange-rate data-sets as follows: If $\varepsilon_{k}=\left\{e_{k}[i]\right\}_{i \in N}$ where $N=\{1,2,3, \cdots\}$ is the time-series of the exchange-rates $e_{k}[i]$ of each country $\mathrm{k}$, out of $\mathrm{m}$ countries in total (i.e. $\mathrm{k}=1,2$, $3, \cdots, \mathrm{m})$, then we define a new set of data $\varepsilon_{k}^{+}=\left\{e_{k}^{+}[i]\right\}_{i \in N}$, where

$e_{k}^{+}[i]=\left(e_{k}[i+1]-e_{k}[i]\right) / e_{k}[i]$,

which is the proportional change in rate over one quarter. We consider these changes to be measures of the resilience of each currency in financial environment. We can then compare two exchange-rates in terms of $\varepsilon_{k}^{+}$to obtain a standardized measure of their relative movement.

This standardized data is plotted in Figure 1 for the period from Q1 2005 to Q4 2012. The extent to which the curves coalesce into a wide band suggests that the average exchange-rate has a similar trend, and this is shown in Figure 2. In both these graphs, we observe unusual behavior in the vicinity of $2008(13 \leq \mathrm{i} \leq 17)$, when most currencies move sharply upward with respect to the USD. The exchange-rates also diverge, which implies that the variance of the distribution of the standardized data when $\mathrm{i}=15$ will also be much higher during this period than in other quarters. 


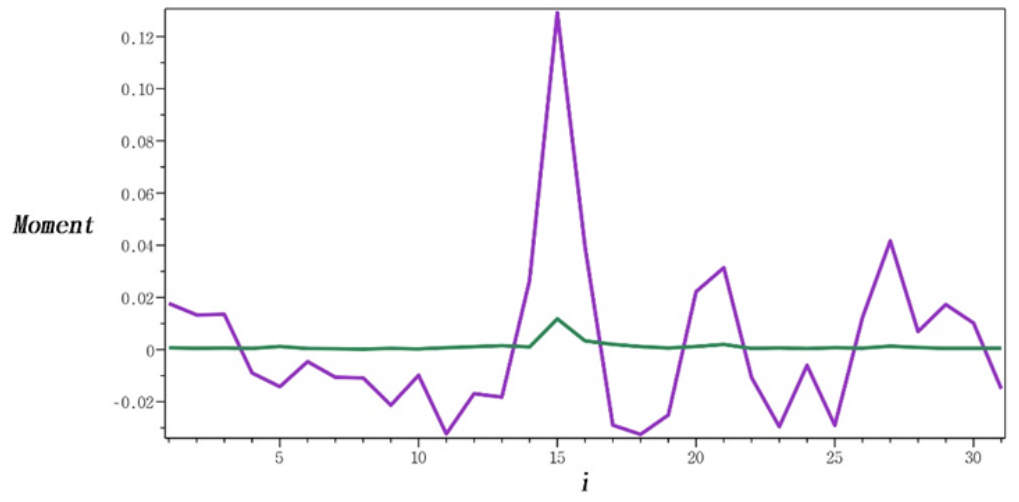

Fig. 2. Mean (purple) and variance (green) of $\varepsilon_{i}^{+}$, for the standardized data-sets of currencies.

In particular, we observe that the EUR and the JPY move in opposite directions, suggesting a relationship in which one of these currencies is able to substitute for the other to some extent. In particular, Japanese yen looks uniquely strong during the crisis, suggesting that it was seen as a safe haven.

We provide support for these assertions in the following subsections.

\subsection{Normalization of standardized data-sets and analysis of higher statistical moments}

Skewness measures the asymmetry of a distribution and kurtosis the sharpness of its peaks and the thickness of its tail. We will now see how skewness and kurtosis behave near the crisis. First, we normalize the standardized data-sets as follows: For $1 \leq \mathrm{k} \leq \mathrm{m}$, let $\varepsilon_{k}^{+}=\left\{e_{k}^{+}[i]\right\}_{i \in N}$ be $\mathrm{m}$ standardized data-sets $\varepsilon_{k}$ of exchange-rates, which we can organize into the following sequence of sets:

$N_{k}^{e}=\left\{Z_{k}^{e}[i] \mid Z_{k}^{e}[i]=\left(e_{k}^{+}[i]-m_{i}\right) / \sigma_{i}\right\}_{i \in N}$

where $m_{i}$ and $\sigma_{i}$ respectively are the mean and standard deviation of $\left\{e_{k}^{+}[i]\right\}_{k \in K}$ at time $i$, for each $k \in K=\{1,2,3, \cdots, \mathrm{m}\}$. The collection of data-sets $\left\{N_{k}^{e}[i]\right\}_{k \in K}$ is the normalization of the standardized data-sets $\left\{e_{k}^{+}\right\}_{k \in K}$, or equivalently the standardized normalization of $\left\{e_{k}^{+}\right\}_{k \in K}$. The standardized normalization of all the exchange-rates that we are considering is shown in Figure 3.

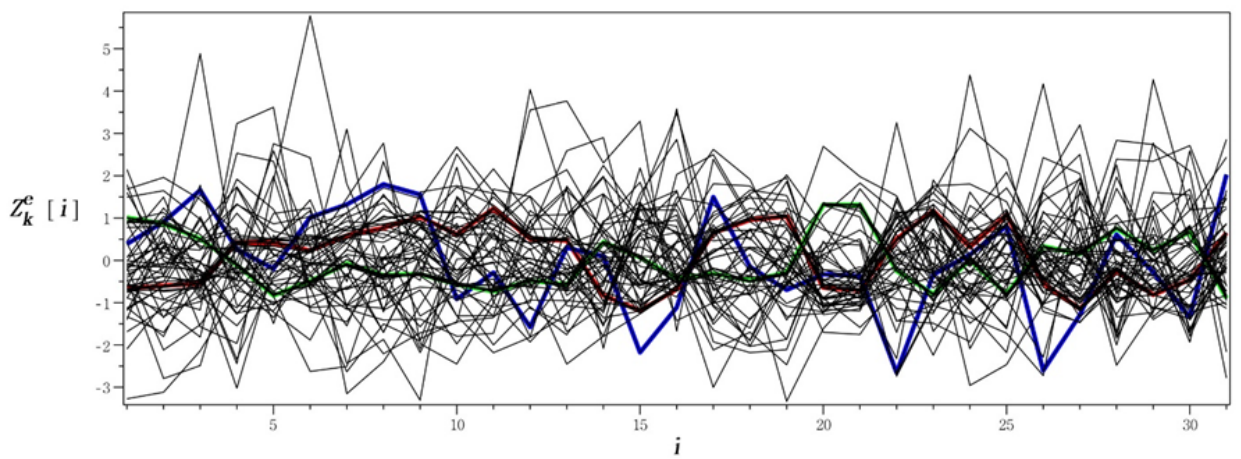

Fig. 3. Standardized normalized exchange-rates. The red, green and blue curves respectively show the USD, EUR and JPY. 
Comparing Figure 3 with Figure 1, we see three significant differences: we can no longer observe the dramatic fluctuation during the crisis $(13 \leq \mathrm{i} \leq 16)$; the relative behavior of the USD, EUR and JPY is different; and the currencies diverge much earlier $(2 \leq \mathrm{i} \leq 7)$ than during the crisis.

To pursue these observations, we perform further analysis of the standardized normalization $\left\{N_{k}^{e}\right\}_{k \in K}$, using skewness and kurtosis, $S^{e}$ and $K^{e}$, which are defined as follows:

$$
\begin{aligned}
& S^{e}[i]=\left(E\left[\left(Z_{k}^{e}[i]-E\left(Z_{k}^{e}[i]\right)\right)^{3}\right]\right) / V\left(Z_{k}^{e}[i]\right)^{\frac{3}{2}} \\
& K^{e}[i]=\left(E\left[\left(Z_{k}^{e}[i]-E\left(Z_{k}^{e}[i]\right)\right)^{4}\right]\right) / V\left(Z_{k}^{e}[i]\right)^{2}-3
\end{aligned}
$$

The time-series of the skewness and kurtosis of the distributions in $\left\{N_{k}^{e}\right\}_{k \in K}$ are shown in Figure 4 .

We see that the fluctuation of skewness and kurtosis is significantly reduced during the crisis (9 $\leq \mathrm{i} \leq 20$ ). This implies that the crisis (especially $\mathrm{i}=14,15$ ) brought the distributions closer to normal distributions, which correlates with the lack of dramatic fluctuations during this period in Figure 3. Kurtosis peaks well before the crisis, between Q2 2005 and Q4 2006 ( $2 \leq \mathrm{i} \leq 8)$, suggesting that it might have functioned as an early indicator.

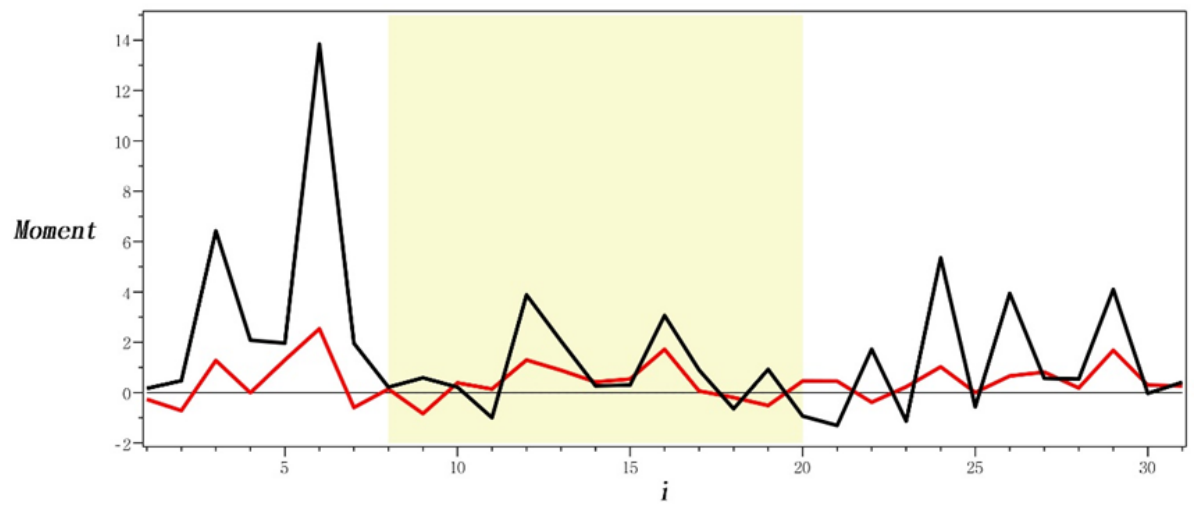

Fig. 4. Skewness $S^{e}$ (red) and kurtosis $K^{e}$ (black) of the standardized normalization of $\left\{\varepsilon_{k}\right\}_{k \in \mathrm{K}}$

\subsection{Analysis on suggestive fluctuations in some exchange-rates}

Next, we will look at the behavior of the USD, EUR and JPY in $\left\{N_{k}^{e}\right\}_{k \in K}$, and also that of the HKD and CNY with respect to the US dollar. If we examine Figure 5 (a), and compare the behavior of the JPY with that of the sum of the USD, EUR and JPY (the broken curve), it appears that the USD and EUR are in a substitute relationship, with the JPY as a secondary substitute. This differs from the conclusions that we drew from Figure 1 . Additionally, wecan now see that, while the USD was weaker than the EUR for a fairly long period $(4 \leq \mathrm{i} \leq 13)$ before the crisis, the USD and JPY became stronger than the EUR, during the crisis $(14 \leq \mathrm{i} \leq 16)$. This suggests that the peak value of kurtosis at Q2 2006 ( $\mathrm{i}$ $=6$ ) that can be seen in Figure 4 indicates the beginning of the long-term USD weakness, which will be discussed further in Section 3.

The relationship between the USD, HKD and CNY in Figure 5 (b) is of interest: while the HKD tracks the USD closely, the CNY closely follows after a short delay. These three time-series almost coincide on seven occasions in the period from the 2008 crisis to the European sovereign debt crisis $(15 \leq \mathrm{i} \leq 21)$. We will now compare the kurtosis of the distributions of exchange-rates and stockmarket indices, with a view to shedding more light on the behavior of exchange-rates near the crisis.

\section{Results}

We now attempt to express the financial state of a country as a function of the exchange-rate of its 
currency and its stock-market index. First, we can standardize and normalize the distribution of stock-market indices in a similar way to exchange-rates. We can then link the standardized normalized exchange-rates $N_{k}^{e}$ and stock-market indices $N_{k}^{a}$ of each country k at each time i to form a pair $\mathrm{p}_{\mathrm{k}}[i]=(x[i], y[i])$, where $x[i] \in N_{k}^{\mathrm{e}}, y[i] \in N_{k}^{\mathrm{a}}$. We take $\mathrm{p}_{\mathrm{k}}[i]$ to be a representation of the financial state of country $k$ at time $i$.
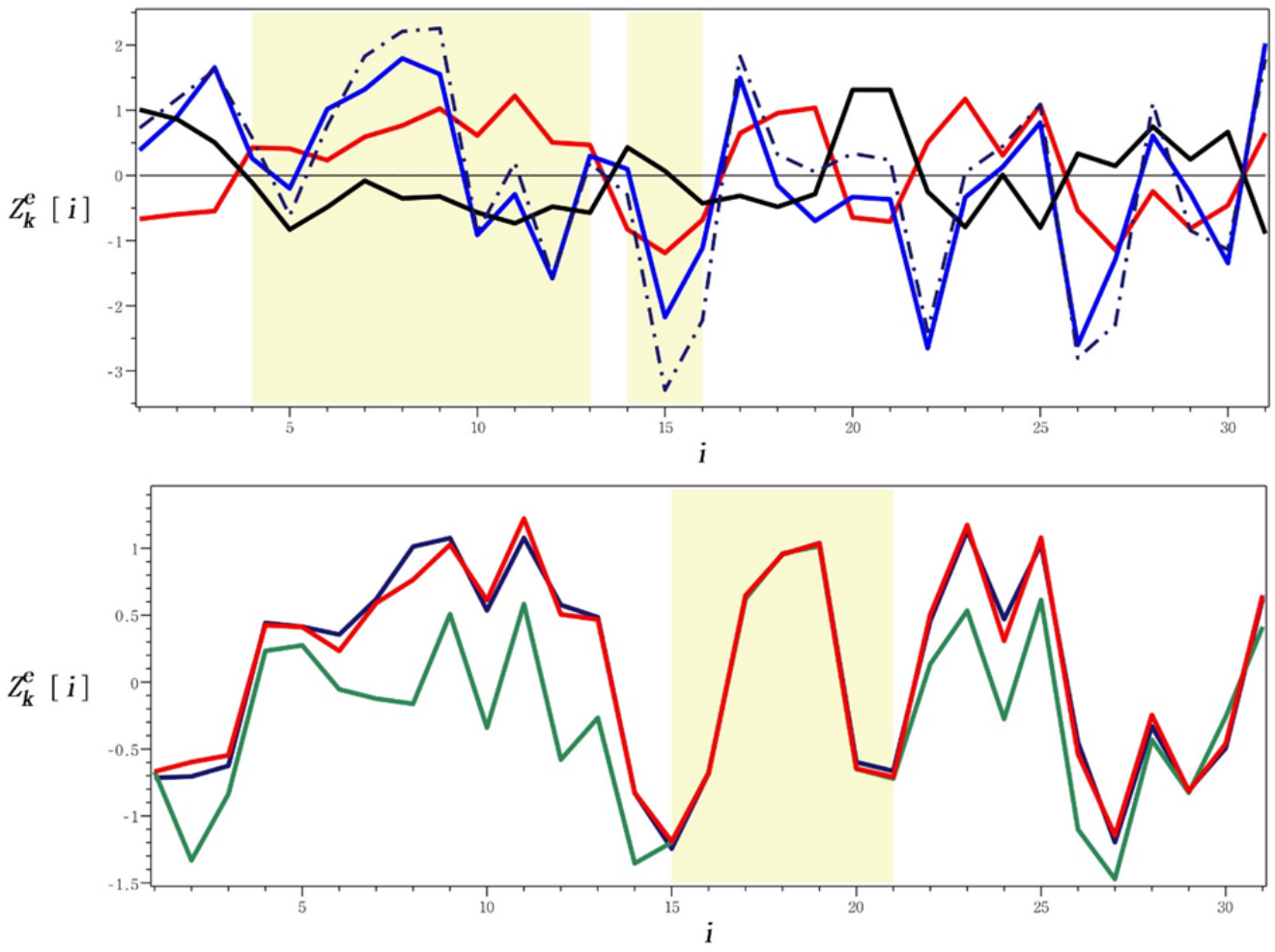

Fig. 5. Comparison of the behavior of some currencies in $\left\{N_{k}^{e}\right\}_{k \in K}$ : (a) the USD (red), EUR (black), JPY (blue) and the sum of the USD, EUR and JPY (broken); (b) the USD (red), HKD (dark-blue) and CNY (dark-green).

Next, we consider the distribution of financial states and its evolution. Figure 6 shows three distributions of the financial states of 51 countries from IMF quarterly figures at Q2 2006, Q4 2006 and Q3 2008: their patterns are significantly different, and we will attempt to characterize these changes over time in terms of the kurtosis of the distributions. Let $\mathrm{K}^{\mathrm{e}}[i]$ and $\mathrm{K}^{\mathrm{a}}[i]$ respectively be the kurtosis of $\left\{Z_{k}^{e}[i]\right\}_{k \in K}$ and $\left\{Z_{k}^{a}[i]\right\}_{k \in K}$. Then we can plot each point $K[i]=\left(K^{e}[i], K^{a}[i]\right)$, with the result shown in Figure 7.

The points K[i] in Figure 7 relate to the differences in the patterns shown in Figure 6 as follows: Both distributions have unusually large values of kurtosis at Q2 2006, which is very different from the behavior of their means. At $\mathrm{Q}_{4}$ 2006, the distribution of exchange-rates has a small value of kurtosis, but the distribution of stock-market indices still has an unusually large value. At $Q_{3}$ 2008, both distributions have small values of kurtosis. We can also see that points with values of kurtosis greater than 6 near the crisis all appear along the y-axis, which suggests that stock-markets were 

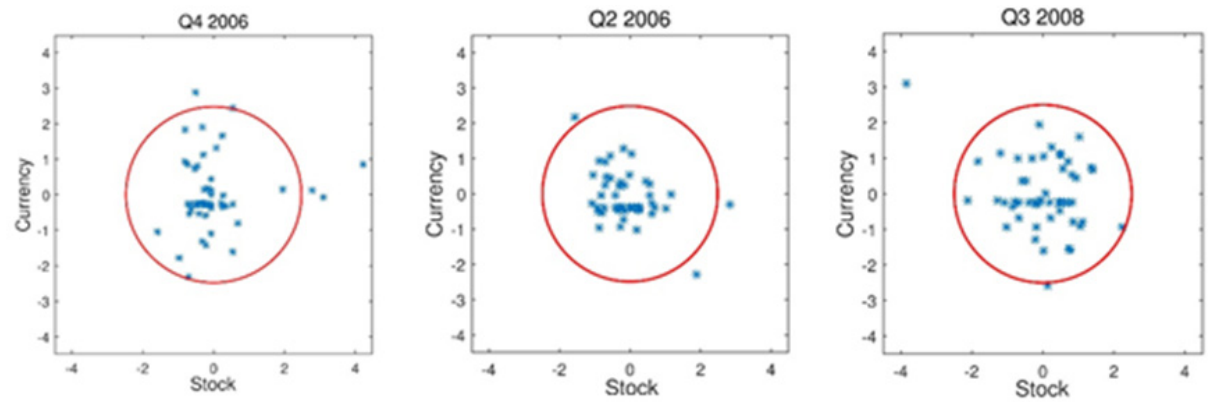

Fig. 6. Distributions of $p_{k}[i]$ when $(a) i=6,(b) i=8$, and (c) $i=15$. The red circle in each sub-figure indicates the confidence circle with a $95 \%$ significance level.

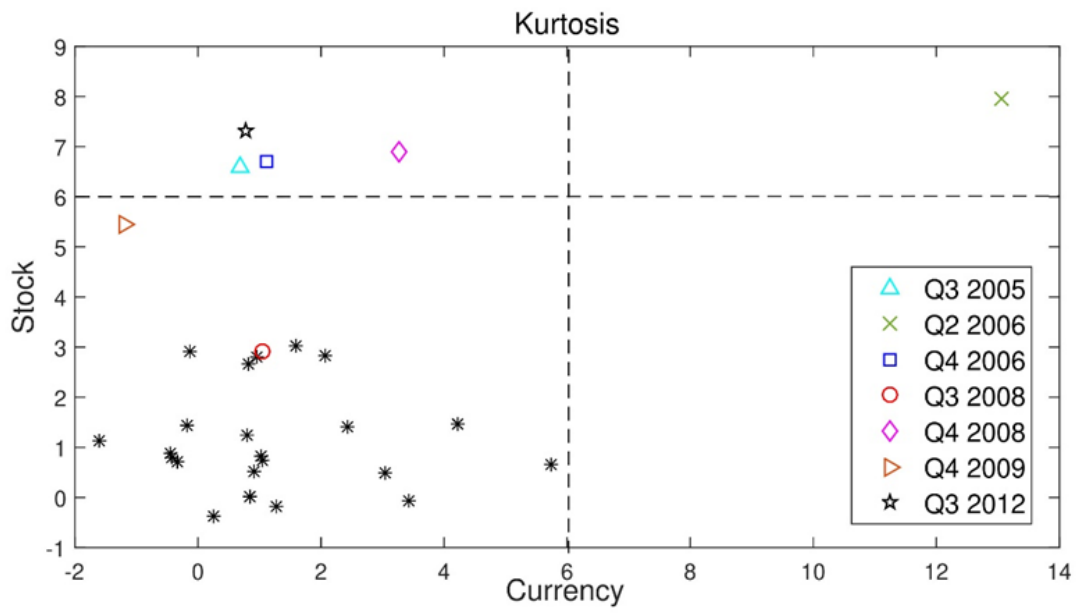

Fig. 7. Distribution of the pairs of kurtosis values $k[i]$ for $1 \leq \mathrm{i} \leq 31$.

responding more sensitively to the crisis than foreign exchange markets. Kurtosis measures the sharpness of the peak of a distribution and the thickness of its tail. Thus the association of the unusually large values of kurtosis of both distributions at Q2 2006 with the start of a long period of USD weakness, suggest that the exceptionally large value of kurtosis of the distribution of exchangerates at Q2 2006 might have functioned as an early warning of the crisis.

\section{Conclusion}

We have examined the behavior of the statistical moments of distributions of the standardized normalization of exchange-rates near the 2008 financial crisis. We have shown that the distribution of exchange-rates had unusual kurtosis before the crisis, with significant fluctuations in the behavior of some currencies.

We compared the kurtosis of the distributions of exchange-rates and stockmarket indices, and their evolution through the crisis. We found that stock markets responded more sensitively to the crisis than foreign exchange markets. We found exceptional values of the kurtosis of both distributions at Q2 2006 which might have signaled the genesis of the crisis. 


\section{Acknowledgement}

This research was supported by Basic Science Research Program through the National Research Foundation of Korea(NRF) funded by the Ministry of Science, ICT and Future Planning (NRF$\left.{ }_{2017} R_{1} E_{1} A 1 A 03070952\right)$ and by the Ministry of Science (2018R1D1A1Bo7051450).

\section{References}

Blanchard, O.J., Das, M., Faruqee, H. (2010). The initial impact of the crisis on emerging market countries. Brookings Pap. Econ. Act. 41, 263-323.

Frankel, J.A., Saravelos, G. (2012). Are leading indicators of financial crises useful for assessing country vulnerability? Evidence from the 2008-09 global crisis. J. Int. Econ. 87, 216-231.

IMF (2015). International Financial Statistics CD-ROM. International Monetary Fund.

Jun, D., Ahn, C., Kim, G. (2017). Analysis of the global financial crisis using statistical moments. Financial Research Letters 21, 47-52.

Lane, P.R., Milesi-Ferretti, G. M. (2011). The cross-country incidence of the global crisis. IMF Econ. Rev. 59, 77-110.

Reuters (2009). Three top economists agree 2009 worst financial crisis since Great Depression; risks increase if right steps are not taken. February 27. Archived from the original on February 12, 2010. Retrieved November $10,2015$.

Rose, A.K., Spiegel, M.M. (2010). Cross-country causes and consequences of the 2008 crisis: international linkages and American exposure. Pacific Econ. Rev.15, 340-363.

Rose, A.K., Spiegel, M.M. (2012). Cross-country causes and consequences of the 2008 crisis: early warning. Japan World Econ. 24, 1-16. 